\title{
Téoros
}

Revue de recherche en tourisme

\section{Le « tout inclus » : contrôle du risque et de la durabilité}

\section{Laura Giobbi}

Volume 31, numéro 2, 2012

URI : https://id.erudit.org/iderudit/1020767ar

DOI : https://doi.org/10.7202/1020767ar

Aller au sommaire du numéro

\section{Éditeur(s)}

Université du Québec à Montréal

\section{ISSN}

0712-8657 (imprimé)

1923-2705 (numérique)

Découvrir la revue

\section{Citer cet article}

Giobbi, L. (2012). Le " tout inclus " : contrôle du risque et de la durabilité. Téoros, 31(2), 12-20. https://doi.org/10.7202/1020767ar

\section{Résumé de l'article}

Dans l'actuelle société postmoderne, les nombreuses options de consommation, la possibilité pour les individus de construire leurs propres biographies en suivant l'impulsion individualiste sans les contraintes de la société traditionnelle, ont inévitablement une incidence sur la pratique du tourisme. Le tourisme contemporain, dit post-tourisme, est aujourd'hui segmenté, flexible et offert sur mesure en fonction des souhaits individuels. Ainsi, la pratique du tourisme " tout inclus " est une variation postmoderne du tourisme de masse, dont les origines dans les années 1920 étaient liées à la sécurité des transports et de l'hôtellerie dans la pratique du tourisme. Cet article illustre comment, dans la période actuelle, le tout inclus prend une signification différente qui répond au climat d'incertitude qui entoure l'individu postmoderne au niveau individuel et collectif. Pleinement conscient de son statut ludique, en payant le voyagiste, le touriste du tout inclus obtient un séjour contrôlé et planifié qui lui permet de vivre une expérience sans risque mais qui rend difficile, en retour, une véritable connaissance mutuelle entre les peuples.

En soulevant les questions liées aux problèmes et paradoxes du tout inclus, cet article questionne la durabilité du concept.
Ce document est protégé par la loi sur le droit d'auteur. L'utilisation des services d'Érudit (y compris la reproduction) est assujettie à sa politique d'utilisation que vous pouvez consulter en ligne.

https://apropos.erudit.org/fr/usagers/politique-dutilisation/ 


\section{Le « tout inclus»: contrôle du risque et de la durabilité}

Laura GIOBBI, Ph.D.

Professeure contractuelle

Université Roma Tre

laura.giobbi@uniroma3.it

RÉSUMÉ: Dans l'actuelle société postmoderne, les nombreuses options de consommation, la possibilité pour les individus de construire leurs propres biographies en suivant l'impulsion individualiste sans les contraintes de la société traditionnelle, ont inévitablement une incidence sur la pratique du tourisme. Le tourisme contemporain, dit post-tourisme, est aujourd'hui segmenté, flexible et offert sur mesure en fonction des souhaits individuels. Ainsi, la pratique du tourisme « tout inclus » est une variation postmoderne du tourisme de masse, dont les origines dans les années 1920 étaient liées à la sécurité des transports et de l'hôtellerie dans la pratique du tourisme. Cet article illustre comment, dans la période actuelle, le tout inclus prend une signification différente qui répond au climat d'incertitude qui entoure l'individu postmoderne au niveau individuel et collectif. Pleinement conscient de son statut ludique, en payant le voyagiste, le touriste du tout inclus obtient un séjour contrôlé et planifié qui lui permet de vivre une expérience sans risque mais qui rend difficile, en retour, une véritable connaissance mutuelle entre les peuples.

En soulevant les questions liées aux problèmes et paradoxes du tout inclus, cet article questionne la durabilité du concept.

Mots-clés: Postmodernité, individualisme, incertitude, durabilité, critique.

Le tourisme tout inclus est le séjour dans lequel la combinaison des services d'hébergement et de transport ou des visites et des excursions guidées est entièrement planifiée par le voyagiste. La naissance de ce type de tourisme date de la propagation du tourisme de masse, à partir des années 1920. Aujourd'hui, le tout inclus est parmi l'une des expressions du tourisme les plus répandues. Pour cette raison, les relations entre les touristes du tout inclus et le territoire d'accueil sont considérables, et les rencontres avec la population locale mettent en cause le concept de «durabilité» du tourisme, en particulièr de sa durabilité sociale.

Le tourisme tout inclus, comme chaque produit touristique, doit être étudié en fonction des différents désirs du touriste. Les désirs sont dépendants de facteurs culturels, sociaux et économiques, fortement influencés par les caractéristiques distinctives de la société à laquelle appartient le touriste. Les exigences que le touriste formule à l'égard de son propre séjour viennent en fait des expériences qu'il ou elle vit dans sa vie quotidienne.
Dans la nouvelle économie de l'expérience, à savoir une économie qui n'est plus basée sur la production de biens et services mais plutôt sur la production et sur le marketing culturel, le tourisme est l'une des nombreuses expériences monétisées que l'individu peut effectuer pratiquement dans le monde entier en payant, pour recueillir des expériences avant de retourner chez lui avec la réalisation de soi. Le tourisme de type «tout inclus» est l'une de ces options.

Cet article analyse la pratique du tourisme tout inclus du côté de la demande formulée par le pays de départ en observant sa signification dans ces sociétés et du côté de l'offre à travers l'étude des conséquences de ce type de tourisme dans les pays d'accueil. Le but de cet article est de savoir si, au-delà du besoin évident de repos de l'individu et au-delà de l'avantage de visiter de nombreux endroits dans un court laps de temps, le tourisme de tout inclus répond à des besoins particuliers de la société actuelle. Comme le tourisme tout inclus ne peut pas être étudié sans analyser ses conséquences sur le pays d'accueil, des questions supplémentaires sont soulevées. Puisque le voyagiste prépare les détails des vacances des touristes de tout inclus, 
comme l'organisation des horaires préfixés des rencontres avec les résidents qui répondent aux différents flux de touristes des divers voyagistes, est-il possible aux touristes de se familiariser avec les gens de la destination hôte? La communauté internationale indique d'utiliser la pratique du tourisme durable pour répondre à ce problème. Toutefois, cette pratique peut-elle, seule, résoudre cette difficulté? Ou le même concept de durabilité n'est-il pas aussi clair qu'il n'y parait?

\section{Le tout inclus : touriste de masse et post-touriste comparés}

Le phénomène du tourisme est étroitement lié aux caractéristiques du temps : ses transformations et son évolution surviennent en relation avec des changements sociaux, politiques et culturels. L'étude des objets typiques recherchés par le regard des touristes peut aider à comprendre les éléments importants de leur société d'origine grâce à sa capacité à révéler des aspects de pratiques sociales courantes. Pour l'analyse du tourisme tout inclus, il est utile de faire une comparaison entre les deux périodes historiques qui ont engendré le tourisme de masse et le tourisme actuel.

Le tourisme de masse s'est propagé aux États-Unis à partir des années 1920 et en Europe depuis la Seconde Guerre mondiale. Il a été appelé tourisme de masse parce qu'il s'est développé dans la société de masse dont il a repris les caractéristiques. Le tourisme de masse pouvait naître seulement lorsque les multiples changements économiques, urbains, infrastructurels et comportementaux du $\mathrm{XX}^{\mathrm{e}}$ siècle ont transformé le mode de vie de la plupart des sociétés européennes.

Ce nouveau type de tourisme débuta donc de ces facteurs principaux. Il faut ajouter la prospérité économique considérable de larges couches de la population qui a mené à l'institution et l'expansion progressive des congés payés aux salariés. La profonde transformation du secteur des transports fut aussi une condition préalable importante. La propagation de l'automobile, de l'avion et du train a contribué à rendre les transits plus rapides et les coûts de vacances plus abordables, commençant le processus de démocratisation des déplacements géographiques.

Le tourisme de masse s'est aussi développé à la fin de la Seconde Guerre mondiale grâce à des changements dans le climat économique et politique, au niveau international.

Du côté de la demande, le tourisme de masse est caractérisé par le nombre élevé de voyageurs qui utilisent les établissements hôteliers. Du côté de l'offre, les éléments du tourisme de masse sont l'insertion des touristes dans un groupe, la standardisation des vacances, les forfaits touristiques mis en place par des entreprises spécialisées (Fink, 1970). Dans le tourisme de masse, la formule tout inclus était très standardisée : au cours des années 1950 et 1960, l'expérience des vacances était programmée considérablement, comme le montrent les études qui examinent les cas anecdotiques où même les repas étaient fixés au début du séjour ainsi que le programme des animations, identiques dans des endroits éloignés les uns des autres (Urry, 1990). D'une période de vacances à l'autre, on proposait les mêmes modèles de divertissement, de régimes alimentaires, de logement et de routine hebdomadaire (Ward et Hardy, 1986).
Le tourisme de masse possède une signification supplémentaire, car il a contribué de façon fondamentale à l'extension de la pratique touristique dans la population de citoyens de pays démocratiques (Alberoni, 1964). Il a aussi été un puissant facteur d'identité nationale et collective (Savelli, 2004). Pourtant, dans les années 1960, la sociologie du tourisme conférait une connotation négative à ce type de tourisme. La raison de cette sous-estimation résidait dans les particularités de la société de masse, qui se caractérisait principalement par la position de passivité de l'acteur social.

Il a fallu attendre les années 1970 pour assister à des changements culturels qui ont contribué à l'émergence d'une pluralité de modes de vie fondés sur la différenciation. Les effets de cette nouvelle culture et contre-culture se reflétaient même dans l'intimité de l'individu, dans ses choix personnels, comme les vêtements, les meubles, la nourriture et même le tourisme. C'est à partir de ce moment que les biens à consommer seront un assortiment de produits qui permettront à l'acheteur de construire son style de vie individuel par des combinaisons personnelles, y compris le choix du tourisme à pratiquer. À la lumière des changements qui ont affecté le tourisme dans l'âge contemporain, on peut voir que le tourisme tout inclus pratiqué dans les années 1950 et 1960 est très différent de celui pratiqué dans la société actuelle dans laquelle, à partir des années 1980, naît le tourisme postmoderne ou post-tourisme (Feifer, 1985).

Aujourd'hui, l'expression tout inclus sous-entend la combinaison préalable de certains éléments : les services d'hébergement, de transport et les visites ou les excursions guidées qui constituent la partie significative de cette pratique (Cardosi et Tripodi, $2011: 238$ ).

Les différences entre la formule tout inclus du passé et celle de l'époque actuelle résident tout d'abord dans les progrès impressionnants en matière de technologie numérique et informatique du $\mathrm{XXI}^{\mathrm{e}}$ siècle. En effet, à la différence du touriste de masse, le post-touriste qui choisit le tout inclus peut décider parmi les différentes offres de forfaits touristiques et parmi de nombreux voyagistes. En effet, les caractéristiques du touriste s'inscrivent aujourd'hui dans le mode typique de la consommation, fondée sur des modèles individuels. La transition de la société de masse à la société des individus (Elias, 1987) a donné lieu à la dimension personnelle et affective de choix dans le large éventail de biens et services disponibles à l'humain postmoderne.

Les nombreuses options de consommation donnent aux individus postmodernes la possibilité de construire leurs propres biographies en suivant l'impulsion individualiste (Beck, 1986). Parmi elles, le tourisme joue un rôle important : les individus conçoivent le voyage comme un facteur de consommation personnelle et autonome leur permettant d'assumer des identités différentes et provisoires selon les endroits où ils sont. La "fièvre de l'ego" implique que les individus des sociétés occidentales ne cessent de poursuivre leur soif d'expérience afin de vivre une vie qui repose entièrement sur des décisions individuelles.

$\mathrm{Si}$ les formes modernes du tourisme de masse ont donc été principalement caractérisées par la standardisation, le tourisme actuel est plutôt segmenté, flexible et produit sur 
mesure : ce n'est plus l'individu qui est modelé sur le tourisme, mais plutôt le tourisme qui est produit en fonction des souhaits individuels, permettant de créer d'innombrables typologies de «néotourismes» (Giobbi, 2009:38). C'est en cela que le tourisme reflète l'individualisme, caractéristique propre de la société postmoderne, dans laquelle les individus peuvent construire leurs propres biographies de choix personnels (Giobbi, 2010b).

Par conséquent, le produit touristique doit être préparé selon les différents types de facteurs de style de vie qui dépendent de facteurs culturels, sociaux, politiques et religieux. Bauman (1999) élabore un "espace esthétique» du tourisme dans lequel chaque consommateur, emporté par ses émotions, peut profiter de ce qu'il veut. Dans ce cadre, le touriste, selon ses goûts personnels, peut décider de l'expérience qu'il veut pratiquer, du lieu dont il veut être séduit comme des sentiments qu'il veut éprouver lors d'une visite. Le but est l'obtention de nouvelles sensations. Cette possibilité de choix rend le touriste du tout inclus pleinement conscient de son statut ludique : le tout inclus devient dans la postmodernité un jeu ou plutôt toute une série de jeux avec de multiples possibilités.

Urry (1990) réfléchit également sur la condition du tourisme comme jeu. Il perçoit le touriste des voyages organisés comme quelqu'un qui joue à l'enfant, comme on le voit dans ses relations avec les "parents-voyagistes» et avec les guides. À cet égard, il écrit : "This is especially clear in guided coach tours. One is told where to go, how long to go for, when one can eat, how long one has to visit the toilet, and so on" (Urry, 1990 : 149). Quand le jeu devient trop réaliste et que le visiteur est désorienté par l'expérience qui commence à dépasser un certain niveau de nouveauté et d'étrangeté, le voyagiste, agissant comme un parent de substitution, crée un microenvironnement de protection, appelé «bulle environnementale» (Boorstin, 1964). Cette bulle s'obtient notamment par la distribution du journal du pays d'origine ou par le style architectural de l'hôtel qui rappelle celui de son milieu d'origine.

Ce jeu joué par les touristes se trouve aussi dans la violation de petits tabous dans différentes formes de consommation, tels que manger ou boire à l'excès, dépenser de l'argent imprudemment, porter des vêtements extravagants, maintenir des rythmes temporels de vie très différents du quotidien. Jafari (1988: 60) observe que le voyage, comme expérience non ordinaire, est de fait de l'ordre du sacré. Il affirme que, quand un touriste part pour ses vacances, il traverse la phase de la séquence d'émancipation, incluant le geste de partir et le sentiment de libération : durant cette séquence, le touriste pratique des activités dans un monde qui est un espace-temps non ordinaire (Jafari, $1988: 64$ ).

Si le développement du tourisme de masse provient de la reconnaissance pour la première fois d'un temps libre aux salariés et de la rationalisation croissante entre le temps de travail et le temps de loisir, au contraire, dans la société contemporaine, le loisir joue de plus en plus un rôle important.

Pour cette raison, les vacances mêmes, le temps d'absence du travail, sont devenues un moment de production symbolique majeur : ayant perdu son identité dans la dimension de travail en raison des changements intenses dans l'emploi, l'individu contemporain construit son identité à travers des choix personnels.

Grâce aux progrès des techniques numériques et informatiques du XXI siècle, le post-touriste peut non seulement voir des paysages très loin en haute définition, mais il peut voir les lieux dans l'instant où il le souhaite, à travers les webcams réparties dans le monde entier. En effet, il existe de nombreux sites consacrés à des forums sur les structures d'accueil qui agissent pour informer les voyageurs sur l'avenir des services et des inefficacités locales.

Par conséquent, une caractéristique fondamentale du post-touriste est qu'il ne doit pas nécessairement quitter ses zones habituelles pour observer des lieux éloignés, contrairement à son ancêtre touriste de masse. Avec la possibilité de récupérer des images, le voyageur peut rester immergé dans sa vie quotidienne et admirer ce qui l'intéresse en grand détail, ce qui lui permet de «voyager en fauteuil». Si, dans la modernité, les lieux des vacances ne pouvaient être joints que par le téléphone ou par les images mises en place par les voyagistes et que le tourisme de masse était l'unique mode pour regarder beaucoup de lieux dans un court laps de temps, aujourd'hui, à travers les yeux des satellites, le touriste peut regarder les lieux et les connaître avant de les visiter, tels qu'il sont tous les jours. Très peu est ainsi laissé à l'imagination et il est donc difficile pour le tourisme postmoderne, et en particulier pour le tourisme tout inclus, de vanter les caractéristiques de l'exploration et de la découverte propre du voyage de l'époque moderne.

\section{Les significations du tourisme postmoderne}

Pour comprendre la place pourvue par le tout inclus dans la postmodernité, il faut analyser d'abord les significations du tourisme dans les théories sociologiques contemporaines. Selon Gottlieb (1982), le but des vacances est essentiellement l'inversion du quotidien, la distinction entre l'expérience ordinaire et inhabituelle. Même dans la réflexion d'Urry (1990), l'élément clé de l'expérience touristique est la différence entre le lieu de la vie habituelle et l'objet de l'observation touristique. Son interprétation de l'expérience touristique se concentre sur l'étude des facteurs de liminalité et d'inversion qui appartiennent à l'expérience du touriste à la recherche d'une expérience extraordinaire. L'étude de l'expérience touristique se révèle de grande importance pour l'analyse des sociétés d'origine. L'étude de la nature des schémas sociaux et culturels du touriste permet d'identifier la réalité inversée et de comprendre ce qui constitue son expérience quotidienne.

Corvo (2005) soutient que l'individu autocentré qui essaie d'atteindre le bonheur vit constamment dans la position d'un touriste, sans jamais s'arrêter pour examiner un rapport et, souvent, ne trouve que des plaisirs immédiats et éphémères. Pour Bauman (1999), le touriste peut être vu comme une métaphore de l'individu à la recherche de son identité, comme la région d'origine n'est plus en mesure de la lui attribuer, c'est-à-dire de générer des significations importantes pour lui. Selon l'auteur, les individus qui sont constamment en mouvement sont des touristes ou des vagabonds (Bauman, 1999 : 189). 
Le degré de mobilité individuelle dépend, en fait, des motivations subjectives, des ressources, des objectifs, des conditions structurelles sociétaires telles que l'adhésion, la stratification et le degré d'ouverture du système social dans lequel l'individu vit. Le tourisme postmoderne représente donc une condition importante pour l'intégration sociale : la liberté de mouvement a une valeur considérable et émancipatrice. À côté de la consommation, elle est l'un des principaux facteurs de stratification sociale.

La mobilité continue de l'individu postmoderne est faite de migrations saisonnières, telles que l'excursion et le tourisme. Elles entraînent Maffesoli (1997) à formuler une sociologie de l'errance. Dans cette théorie, le touriste est considéré comme une personne qui incarne le «désir d'ailleurs», complètement tourné vers «l'échappée belle». Pour Maffesoli (1997 : 143), le mouvement physique incarne aussi une errance cognitive parce qu'il produit l'expérimentation de nouvelles réalités relationnelles.

Le paradigme des nouvelles mobilités (new mobilities paradigm, Sheller et Urry, 2006) considère le tourisme comme exemple paradigmatique de la transformation des sites en tant que nœuds mobiles dans l'intersection des flux et des réseaux de la mondialisation. La sociologie des mobilités (Giobbi, 2010a) est basée sur l'hypothèse que l'expérience sociale contemporaine est entourée par des connexions multiples et étendues, organisée autour d'un complexe réseau de mobilités physiques et symboliques dans lesquelles les gens vivent les relations avec les autres. Cette nouvelle discipline étudie les relations sociales des mobilités qui ont donné un nouveau sens à la société. Parmi ses objets d'étude, au sein des migrations saisonnières, les mobilités touristiques jouent un rôle important (Giobbi, 2010 b), et le nombre de personnes impliquées a tendance à augmenter, malgré le ralentissement économique et en raison des nombreuses conséquences que les mobilités touristiques produisent dans les lieux d'arrivée. Le tourisme, qui est l'expression pure de la société mobile contemporaine, devient le phénomène clé pour comprendre la société postmoderne.

À partir de ces théories qui encadrent le tourisme dans la société actuelle, à l'intérieur de ses éléments propres, on peut saisir le sens premier du tout inclus d'aujourd'hui.

\section{Le tout inclus, réponse à l'incertitude postmoderne} L'individu postmoderne a à sa disposition un large éventail de moyens avec lesquels vivre sa mobilité touristique. Les caractéristiques de cette mobilité correspondent à celles de la société actuelle. Parmi tous les choix potentiellement réalisables, il y a deux polarisations des pratiques touristiques actuelles : le tourisme avec destination à surprise et le tourisme tout inclus.

Dans le tourisme avec destination à surprise, pour lequel de plus en plus d'agences de voyages sont en train de se spécialiser, les touristes confient le choix de la destination au voyagiste et sont informés du lieu de leurs vacances seulement quelques jours avant le départ, comme si le touriste était incapable de choisir parmi les nombreuses expériences disponibles. Ce type répond à l'exigence du post-touriste de chercher constamment de nouveaux éléments et de nouvelles sensations. Ici, le voyage est la recherche pure de l'extraordinaire, de la différence par rapport à ce qu'on vit toujours dans le quotidien.
Opposé à cette expérience est le tout inclus. Le choix du tout inclus reflète les caractères de la société postmoderne. En particulier, ce type de tourisme ne répond pas seulement au besoin du touriste de se déstresser ni au besoin du voyagiste d'organiser le voyage pour beaucoup de touristes en optimisant le temps, mais l'hypothèse de l'auteure est que, dans la société postmoderne, il témoigne du climat d'incertitude qui entoure l'individu.

Si, dans le territoire d'origine, la peur est combattue avec la fortification de l'espace privé, avec des portes de plus en plus verrouillées et des systèmes de sécurité, dans les zones touristiques, la peur des dangers qui peuvent être présents dans la région visitée est évacuée par la pratique du tout inclus, qui est tout à fait semblable et représente un moyen de défense personnel prévu par le touriste. Dans ses excursions, le touriste du tout inclus, en fait, reste pour la durée du voyage dans le groupe sans devoir prendre des décisions, protégé et séparé du monde extérieur. Ce type de tourisme insère le touriste dans un programme établi en détail et, dans certains cas, il comprend un séjour dans les aires protégées où le touriste est gardé et, dans le même temps, séparé du monde extérieur. Ici, à l'exception des sorties organisées, il peut aussi vivre fermé dans des villages créés pour ce besoin; la composition et la même architecture de ces villages sont très différents des lieux environnants, ils ressemblent à des quartiers résidentiels urbains protégés.

Le tourisme tout inclus est l'une des expressions les plus courantes du tourisme et ressemble aux premières mesures prises par le tourisme moderne, où les touristes, dépourvus de connaissances nécessaires pour faire face seuls à l'expérience du voyage, se reportaient à l'expérience des guides pour avoir la sécurité d'un séjour bien planifié. Face à une très bonne organisation de ses vacances par les voyagistes, les touristes obtiennent un séjour hyper contrôlé qui leur permet de vivre des expériences sans risque. Cette pratique est née parce que la certitude, la sécurité et la rigidité représentaient les éléments du monde moderne autour desquels la société était officiellement organisée. Au contraire, la réalité sociale actuelle est composée de nouvelles structures qui ont conduit à la naissance de la société postmoderne et qui perturbent l'individu.

Ces nouvelles structures sont liées tout d'abord aux phénomènes de la mondialisation, le processus qui s'est développé en parallèle avec la crise de l'identité nationale. Grâce à la mondialisation, l'individu et ses actions sont placés dans des réseaux transnationaux de dépendance mutuelle, le long de l'axe local-global, dans le monde entier. La déterritorialisation de la base territoriale de l'État, ainsi que la compression de l'espace et du temps, rapprochent tout un chacun et facilitent la rencontre avec l'Autre, mais en même temps exposent les sociétés liées à des dangers constants. La rapidité et l'immédiateté des médias électroniques et des nouveaux médias, qui sont les conditions essentielles de la mondialisation, annulent l'espace et arrêtent le temps : tous les événements indésirables qui se passent partout dans le monde ont des répercussions immédiates sur l'individu, produisant en lui des sentiments d'anxiété amplifiés par les mêmes médias qui diffusent en continu des signaux de danger. 
De plus, les dispositions nouvelles proviennent des profonds changements dans le domaine du travail. D'ailleurs, il faut souligner le facteur croissant d'insécurité : l'emploi n'est plus garanti par la poursuite d'un certain niveau d'études et l'actuelle économie internationale est en crise. Cela provoque de nombreuses conséquences dans la société, car l'absence d'un salaire stable conduit à une autonomie retardée et, par conséquent, à la difficulté de former de nouvelles familles. À cela, il faut ajouter la décomposition des rôles familiaux traditionnels.

Parmi les processus qui ont conduit à l'émergence de la société actuelle, il y a la perte de l'aspiration, propre de l'ère moderne, pour trouver un seul sens du monde à partir de principes métaphysiques, idéologiques ou religieux (Lyotard, 1979). La nouvelle scène sociale postmoderne, appelée "société du risque» (Beck, 1986) ou «société de l'incertitude» (Bauman, 1999), a modifié les structures de pensée et de vie des individus. Dans la société d'aujourd'hui, les instances de liberté et de sécurité vont de pair : si l'absence de références solides a permis d'un côté la croissance de la liberté personnelle, de l'autre côté, elle a affaibli l'individu.

$\mathrm{Au}$ niveau macrosocial, les principales raisons de l'insécurité postmoderne peuvent être retracées dans la présence d'un «new world disorder» (Jowitt, 1992), qui se présente sans structures préétablies et sans une logique compréhensible. Dans la déréglementation universelle, la priorité donnée à la concurrence sur le marché cause une lacération sociale de tous les filets de sécurité et au mépris de toutes les raisons non économiques. À ces macrocauses, il faut ajouter le scepticisme à l'égard des institutions qui ont traîné l'individu dans l'expérience de la guerre et qui souvent aujourd'hui sont accusées d'être devenue une élite qui abuse du pouvoir conféré par le peuple à des fins personnels, au lieu de poursuivre le bien commun.

Au niveau microsocial, les préoccupations de la société contemporaine sont délimitées d'une manière différente que par le passé : l'inquiétude place l'individu dans un état d'incertitude, d'insécurité existentielle. Même les risques qui menacent sa santé sont configurés différemment que dans les temps modernes. Effectivement, ils sont en mesure de se soustraire à la perception immédiate, comme cela se produit pour les substances radioactives ou toxiques qui sont présentes tous les jours dans l'air, l'eau et la nourriture, entraînant des dommages dans le long terme. Cet état d'anxiété constante fait sensiblement augmenter la demande pour la protection des citoyens, conduisant à d'importantes externalités dans les domaines social, économique, politique et touristique. La peur de la délinquance dans la société d'accueil reflète les angoisses postmodernes sur le plan personnel, à savoir l'absence de garanties de sécurité pour sa propre personne et l'insécurité causée par la perte de confiance dans les intentions des autres. Souvent, garder l'autre à distance est devenu une stratégie de survie.

Dans un tel climat d'insécurité qui entoure l'individu, le rôle du tout inclus est considérable pour répondre à la demande de garanties de sécurité en vacances. En payant le voyagiste, le touriste obtient un séjour contrôlé et planifié qui lui permet de vivre une expérience sans risque.

\section{La durabilité du tout inclus dans les relations avec les résidents}

La fortification du touriste dans le tout inclus contribue à établir une relation superficielle avec la société d'accueil et rend plus difficile de répondre à la durabilité sociale du territoire.

La relation qui se développe entre les touristes et la population résidente est caractérisée par certaines conditions. Il s'agit d'une expérience transitoire qui a des limites spatiales et temporelles, qui manque de spontanéité et qui peut conduire à des conditions d'inégalité et de déséquilibre entre les sujets prenants. Par conséquent, des destinations, au début balnéaires, se sont développées jusqu'à offrir des structures d'hébergement qui répondent à tous les besoins des vacanciers, dans des lieux ressemblant de plus en plus à des enclaves, loin de la population locale. Cette ségrégation des touristes dans les destinations d'accueil arrive souvent dans des pays en développement, qui souffrent de la pauvreté et du manque de ressources. Dans un tel espace social, les relations entre les touristes et les populations locales sont entièrement déterminées et planifiées par les voyagistes.

Pour MacCannell (1976), les touristes sont en quête d'authenticité et donc ils recherchent des formes d'interaction avec la population locale, qui à son tour considère les visiteurs comme une partie de leur environnement. Dans cette réflexion, MacCannell (1976 : 590) applique au tourisme les notions de front region, l'avant-scène, et de back region, l'arrière-scène, développées par Goffman (1959). Il soutient que le lieu où se rencontrent officiellement le touriste et la communauté locale est l'avant-scène. Le lieu où la population se repose et mène sa vie normale, quand elle n'est plus contrainte par les représentations pour les touristes, est l'arrière-scène.

Alors que les touristes veulent entrer dans la zone pour vivre une véritable expérience de contact avec les gens et les cultures autres, il est vrai que les zones touristiques de l'avant et de l'arrière-scène sont difficiles à distinguer. En effet, ces régions sont souvent artificielles, élaborées dans le seul but de satisfaire une représentation de l'authenticité volontairement construite. Entre la scène et l'arrière-scène, il y a des positions intermédiaires qui représentent les étapes à suivre pour atteindre la relation authentique avec la population locale. En plus de l'avant-scène où la zone dans laquelle les relations avec les touristes sont entièrement déterminées par l'organisation du tourisme, il y a : l'avant-scène masquée par quelques détails de l'arrière-scène, dont le but est d'évoquer l'atmosphère du lieu; l'arrière-scène simulée et entièrement organisée pour les touristes; l'arrière-scène ouverte aux touristes alternatifs qui, en suivant des chemins particuliers, peuvent accéder à l'arrière-scène; l'arrière-scène modifiée où l'accès est possible à tous, mais seulement pour un regard marginal; et enfin, l'arrière-scène elle-même, l'espace social qui forme la conscience touristique. Donc, les espaces touristiques sont bien organisés autour d'une «mise en scène d'authenticité ».

Contrairement à l'élaboration de MacCannell (1976), Cohen (1974) a porté son discours de la relation entre les touristes et la population parmi les touristes et l'organisation du tourisme, où la population locale constitue le contexte environnemental du rapport. Sur la base de sa réflexion, le tourisme tout inclus ne se décline pas alors dans la situation 
d'authenticité représentée dans laquelle l'organisation du tourisme prépare l'authenticité simulée que le touriste ignore, mais dans le tourisme planifié, c'est-à-dire quand la scène est entièrement mise en place par l'organisation du tourisme avec la pleine conscience du touriste.

Par contre, au centre de la pensée sociologique d'Urry (1990), il y a le regard du touriste, à savoir la «consommation visuelle» des lieux visités, qui n'est pas valable pour toutes les sociétés à tout moment, mais qui varie en fonction de la société, du groupe social et de la période historique.

Toutefois, le contact avec les peuples locaux à travers le tourisme tout inclus produit une connaissance superficielle de la population locale. En outre, le touriste, séparé du monde extérieur, ne s'intéresse pas aux problèmes de la société d'accueil avec laquelle il ne peut instaurer une relation véritable. Le tourisme tout inclus prend une position antithétique au tourisme durable, inverse à l'expérience touristique, et s'approche de la théorie du sight seeing des années 1960 qui met l'accent sur les aspects les plus superficiels et aliénants du tourisme. Selon cette approche, les touristes ne cherchent pas les choses dans leur réalité, mais recherchent leur représentation, en réduisant la même chose à de simples images (Burgelin, 1967). La rencontre entre le résident et le touriste se produit dans un contexte de pure fiction, dans lequel la réalité d'accueil est prête pour le touriste et est réduite à des images stéréotypées, perdant ainsi sa véracité.

Contrairement aux réflexions de MacCannell (1976) sur la recherche d'authenticité poursuivie par le touriste, le touriste peut aussi apprécier la non-authenticité de l'expérience touristique et il peut s'amuser grâce au grand nombre de distractions créées spécifiquement pour lui. Cette perception, la vision artificielle, est caractérisée par le fait que l'expérience du touriste à la recherche de l'exotisme à tout prix s'arrête à une relation superficielle avec le pays de la destination. La réalité de la destination est mise en place pour les touristes et réduite à des images créées spécialement pour lui, au point de perdre à la fin sa véracité. Ce traitement se réfère à tous les événements touristiques totalement déracinés de leur contexte culturel et social, faits uniquement pour le divertissement des touristes. Cela arrive, par exemple, pour les représentations des cérémonies traditionnelles ou des rituels pour la dramatisation des événements, effectuée selon un programme et à des moments prédéterminés, par exemple dans les moments de rencontre avec la faune locale, avec la présence de «certains lions du Kenya, fidèles au rendez-vous fixé chaque soir par une habile guide» (Augé, 1997 : 97). Ainsi, souvent les particularités des lieux sont stéréotypées et réduites à des étiquettes pour attirer les touristes.

Dans la pratique du tout inclus, l'imprégnation, l'établissement d'une relation naturelle avec la population d'accueil, la découverte comme une forme de connaissance et, enfin, l'aventure, c'est-à-dire le voyage effectué avec une immersion totale dans la réalité d'accueil, deviennent très difficiles à suivre.

La première étape vers la durabilité touristique locale et la conséquente utilisation du tourisme comme instrument de compréhension entre les différentes cultures, c'est d'observer les dynamiques sociales qui se développent dans les relations hôtes-invités. Beaucoup de sociologues (entre autres Smith, 1977; Pearce, 1987; Nocifora, 1996; Savelli, 1998 ; Swarbrooke, 1999) se sont penchés sur ce facteur et sont arrivés à la conclusion que l'impact social du tourisme sur les communautés d'accueil est directement proportionnel à la différence entre les diverses cultures des populations, notamment en matière de symboles, de langue, de valeurs et de règles qui constituent les éléments propres à chaque culture en les distinguant les unes des autres.

Les services du pays d'accueil sont emballés pour les touristes, ce qui rend difficile d'établir des relations authentiques avec les habitants. En fait, il est indubitable qu'il est malaisé de promouvoir une rencontre fructueuse entre les peuples en termes de compréhension (comme le dit l'article 1, premier alinéa, des principes du Code mondial d'éthique du tourisme, OMT, 1999) et d'éviter la standardisation des produits artisanaux et culturels traditionnels (article 4, quatrième alinéa) lorsque le voyage des touristes et les rencontres locales sont établis dans les détails à l'avance et qu'ils s'arrêtent à capturer l'apparence des lieux et des peuples d'accueil.

Ainsi, le voyage n'est plus une occasion de véritable expérience qui permet d'encourager le touriste à la recherche d'un "égard authentique», visant à un contact réel avec la culture locale. Entre la population locale et les touristes, les tensions et les conflits prennent les places réciproques de la connaissance et de la compréhension.

\section{Les questions critiques et les paradoxes du tourisme durable}

La communauté internationale indique deux principaux outils pour surmonter le problème de l'impact du tourisme sur la population locale : le tourisme responsable et le tourisme durable. Le premier est utilisé comme outil de compréhension et de paix minimisant l'impact social sur la communauté d'accueil. Le second vient de la notion de développement durable (UNEP : 1987). Le tourisme durable est un tourisme qui prend en compte les besoins des touristes et des régions d'accueil tout en maintenant l'intégrité culturelle, la biodiversité et les équilibres fondamentaux de la nature, et qui vise à améliorer la qualité de vie de la population locale, avec la protection et l'amélioration des possibilités pour l'avenir.

Bien que les définitions de développement et de tourisme durable énoncées contiennent de bonnes intentions, toutefois, il faut souligner que le concept de durabilité apparaît faussement limpide. En fait, si le concept est démontré très clairement, à savoir que toute action humaine doit être réalisée dans les équilibres dynamiques d'un système, effectivement, il cache de nombreux malentendus et de sombres questions.

Tout d'abord, il faut noter qu'il est extrêmement difficile, presque impossible, d'être sûr qu'une action peut être adéquatement soutenue par le système naturel sur lequel elle est accomplie en raison de l'absence de connaissances objectives et de l'incontestable présence de conséquences involontaires, extrêmement difficiles sinon impossibles à prévoir.

En fait, la complexité du fonctionnement des systèmes naturels rend impossible la connaissance a priori des conséquences de l'action humaine. On ne peut appliquer la relation linéaire pour laquelle à chaque cause (l'action de l'homme) 
est associé un certain effet. En plus, cette certitude ne serait même pas suffisante, car le contrôle devrait être fait d'une fois à l'autre, en cours de route (Bologna, 2005). Il convient également de noter que le principe de la durabilité, s'il n'est pas appliqué aux caractéristiques du cas spécifique, est susceptible de rester une base abstraite qui, dans la pratique, est très difficile à suivre.

L’ambiguïté de la définition du développement durable a été l'objet de critiques dans la même période de sa formulation, de sorte que, en 1991, une autre définition a été développée dans le rapport intitulé Caring for the Earth (UICN, PNUE et WWF, 1991). Ce document, préparé par le Programme des Nations Unies pour l'environnement (PNUE), l'Union internationale pour la conservation de la nature (UICN) et le Fonds mondial pour la nature (WWF), était le noyau de la nouvelle définition qui vise à améliorer la qualité de vie des hôtes en restant dans les limites de la capacité de charge des écosystèmes terrestres.

La distinction entre les deux définitions réside dans la différence conceptuelle entre le développement durable et la durabilité du développement. La première expression est placée dans une perspective anthropocentrique et peut être insérée dans la logique de l'écologie superficielle (shallow ecology, Naess, 1973). Avec cette expression, on assigne un rôle passif à l'environnement, de simple usage humain, en établissant le système économique et financier dans une position de priorité sur le système politique et écologique, de sorte que, dans ce cas, on devrait parler d'une croissance durable. Au contraire, la deuxième définition implique une perspective biocentrique, car elle établit la priorité de la situation écologique et politique en promouvant la réduction de la consommation et un mode de vie caractérisé par la sobriété, c'est-à-dire la réalisation de l'écologie fondamentale (deep ecology).

Une autre critique de la notion vient de Sen (1999) qui interprète le concept de développement durable, au sens donné en 1988, dans la perspective des habitants les plus pauvres de la planète. Il questionnait la signification pour ces gens du concept de développement durable, qui signifiait pour eux la garantie dans l'avenir d'une qualité de vie égale à celle présente.

Avec cette analyse on comprend que les véritables présupposés de la durabilité résident dans la coopération au niveau mondial, assurant une vie digne pour tous les habitants de la planète. Parallèlement à la notion de durabilité il faut prendre soin des besoins individuels de santé et de bien-être, de nourriture et d'abri adéquat, d'expression et d'identité culturelle. La relation entre le tourisme et l'environnement est purement circulaire, car il y a un lien indissociable entre le tourisme et l'environnement physique et social.

La propagation de l'empreinte humaine arrive sur une grande partie de la surface de la terre, au point que, selon de nombreux scientifiques, il n'y a pas de zones terrestres qui n'aient reçu l'influence de l'être humain. Par conséquent, il n'est pas possible de parler aujourd'hui de lieux intacts. À cet égard, paradoxalement, il arrive souvent que l'expérience touristique est racontée dans le soi-disant "syndrome d'Armstrong", ce qui se produit lorsque les touristes considèrent leurs actions à l'égal de ceux du premier humain sur la lune, en répétant, à l'occasion de l'histoire des vacances : « Sono stato il primo a...», «Pensa che non avevano mai visto un...» («J'ai été le premier à...», "Je pense qu'ils n'avaient jamais vu un...», traduction libre de l'auteure), (Canestrini, 1998 : 331).

Le paradoxe du tourisme provient de la relation circulaire entre le tourisme et l'environnement. D'un côté, le tourisme est possible grâce aux ressources et aux cultures locales, puisque l'environnement est la condition essentielle du tourisme. De l'autre, les activités touristiques ont un tel impact sur la zone qu'elles arrivent à détériorer et même à détruire ces ressources, en causant la diminution de l'attractivité du lieu et en générant, par conséquent, l'épuisement et la disparition des touristes eux-mêmes.

Cependant, dire que le tourisme contient en lui-même les éléments de sa destruction, c'est une déclaration trop étroitement liée à la logique du fonctionnalisme (Parsons, 1937) pour laquelle, si l'acteur (dans ce cas, le touriste) d'un système (dans ce cas, l'environnement local et l'industrie du tourisme) est intégré dans le système, son action est fonctionnellement positive. Inversement, elle est dysfonctionnelle, fonctionnellement négative, lorsque le système touristique serait inefficace à répondre à l'impératif fonctionnel de l'intégration, précisément en raison de son acteur, le touriste.

Parmi les critiques adressées au paradoxe du tourisme, l'observation de Knafou (1998:39) est intéressante. Il affirme que la théorie selon laquelle le tourisme est autodestructeur pour sa propre nature est en effet un cliché, un dommage courant dans la littérature du tourisme. Pour Knafou (1998: 42), cette analyse est fondée sur deux hypothèses contestables : l'opinion largement répandue que ce qui est arrivé dans la période passée est toujours mieux que ce qui arrive aujourd'hui et la mauvaise considération du rôle touristique des patrimoines naturels.

La première hypothèse est utilisée souvent, il s'agit de la logique d'une excessive mise en valeur du passé. Selon cet argument, la destination était toujours plus belle avant l'arrivée des touristes, qui mettent continuellement en danger l'harmonie et le charme des lieux. La deuxième hypothèse se rapporte à une réflexion sur les ressources. Ce qui est critiqué, c'est la théorie selon laquelle il existe une ressource en ellemême qui serait suffisante à découvrir, développer et promouvoir. Au contraire, l'auteure observe comment l'attractivité d'un lieu est en fait une invention d'une certaine société à un certain moment. Knafou (1998:41) affirme que les côtes, les montagnes ne furent pas toujours attrayantes et ne le seront pas toujours parce que l'attrait des ressources naturelles dépendent de la société et de l'époque d'appartenance. Si, par exemple, dans le passé, la campagne était le paysage le plus commun et la vie rurale était jugée difficile et nécessaire à la survie, maintenant, elles sont devenues des ressources touristiques, comme le reflète la croissance du tourisme agricole et des villages.

\section{Conclusion}

Cet article a tenté de saisir les éléments du tourisme de masse et du tourisme postmoderne en présentant leur signification. Afin de comprendre les différences avec le tourisme tout inclus contemporain, l'auteure a analysé les significations 
du tourisme dans l'âge postmoderne. De cela a découlé l'explication que le tourisme tout inclus dans la société actuelle satisfait un besoin particulier différent du passé, car il est la réponse au climat d'incertitude qui entoure l'individu postmoderne au niveau individuel et collectif.

La dominante perception du risque influence aujourd'hui les acteurs sociaux afin de les diriger vers un usage touristique qui minimise ses préoccupations et ses angoisses. $\mathrm{Si}$, sur son territoire d'origine, la peur de l'Autre est combattue avec la fortification de l'espace privé, dans les zones touristiques, cependant, la même crainte est enlevée par la pratique du tout inclus. Le forfait qui comprend transferts, hébergement, restauration, visites touristiques et divertissements en fonction des besoins du client permet de garder à distance les problèmes et les risques du pays d'accueil et de bénéficier de la réalité construite spécifiquement pour le touriste.

Toutefois, en répondant à la question de la sécurité, le tout inclus prend souvent la forme d'un tourisme qui ne respecte pas les lieux visités. Le détachement ou le contact avec les indigènes produit une connaissance superficielle de la population locale. En restant séparé du monde extérieur, ce type de touriste parvient difficilement à établir une relation authentique avec les populations locales.

Tout en répondant à la question de la sécurité individuelle, le tourisme tout inclus, donc, provoque de nombreuses conséquences sociales sur la population locale. On n'a pas l'intention de condamner ici le tourisme tout inclus ou de donner de jugements moraux, en le considérant comme un type de pratique barbare perpétré dans les territoires d'accueil. Cette pratique permet aux gens d'optimiser le temps et, comme chaque expérience touristique, c'est toujours un facteur de croissance personnelle, car le voyage vient de la volonté de vivre une expérience en rencontrant populations, cultures et lieux, en obtenant un enrichissement intérieur.

Néanmoins, le contact avec les peuples autochtones à travers le tourisme tout inclus produit une connaissance superficielle de la population locale, plus dictée par la curiosité ou le gain de temps que par un véritable intérêt dans la rencontre avec l'Autre.

C'est de cette manière que le tourisme tout inclus ne permet pas d'établir une véritable relation avec les gens autochtones. Dans le tourisme tout inclus, l'imprégnation, c'est-à-dire l'établissement d'une relation avec la population locale, la découverte comme une forme de connaissance et, enfin, l'aventure, à savoir le voyage effectué par une immersion totale dans la réalité d'accueil, devient presque impossible.

Alors, le voyage n'est plus une opportunité qui encourage les touristes à chercher un «égard authentique» qui vise à un contact réel avec la culture locale : entre la population d'accueil et les touristes, la connaissance et la compréhension mutuelle sont rendues plus difficiles.

Si d'un côté la communauté internationale indique comme solution à ce problème la pratique d'un tourisme durable, toutefois, comme l'auteure l'a soutenu, son application n'est pas toujours aussi linéaire qu'il le semble. Le même concept de durabilité n'est pas aussi clair qu'il y parait, mais il cache de nombreux malentendus et de sombres questions qui confinent le concept dans le domaine prescriptif plutôt qu'applicatif.

\section{Références}

ALBERONI, Francesco (1964) Consumi e società, Bologna : Il Mulino. 303 p.

AUGÉ, Marc (1997) L'impossible voyage. Le tourisme et ses images, Paris : Éditions Payot \& Rivages. 187 p.

BAUMAN, Zygmunt (1999) In search of politics, Cambridge : Polity Press. $212 \mathrm{p}$.

BECK, Ulrich (1986) Risikogesellschaft: auf dem weg in eine andere moderne, Subrkamp Verlag, Frankfurt am Main; traduction française (2001) La société du risque. Sur la voie d'une autre modernité, Paris : Édition Aubier. $391 \mathrm{p}$.

BOLOGNA, G. (2005) Manuale della sostenibilità. Idee, concetti, nuove discipline capaci di futuro, Milano : Edizioni Ambiente. $331 \mathrm{p}$.

BOORSTIN, Daniel J. (1964) The image: a guide to pseudo-events in America, New York : Harper. 315 p.

BURGELIN, Olivier (1967) «Le tourisme jugé», Communication, n 10, p. 65-96.

CANESTRINI, Duccio (1998) L'insostenibile retorica del Paradiso, DANS ZERBI, Maria Chiara (sous la direction de), Turismo sostenibile in ambienti fragili, Milano : Cisalpino. 599 p.

CARDOSI, Gianfranco et Enzo M. TRIPODI (2011) Il Codice del turismo, Sant'Arcangelo di Romagna : Edizioni Maggioli. 342 p.

COHEN, Erik (1974) «Who is a tourist? A conceptual clarification », The Sociological review, vol. 22, $\mathrm{n}^{\circ}$ 4, p. 527-555.

CORVO, Paolo (2005) I mondi nella valigia. Introduzione alla sociologia del turismo, Milano : Vita e Pensiero. 144 p.

ELIAS, Norbert (1987) Die Gesellschaft der individuen, Suhrkamp Verlag; traduction française (1991) La société des individus, Paris : Fayard. 316 p.

FEIFER, Maxine (1985) Going places. The ways of the tourist from Imperial Rome to the present days, London : Macmillan. $288 \mathrm{p}$.

FINK, Christian (1970) Der Massentourismus, Berne : Verlag Paul Haupt. $217 \mathrm{p}$.

GIOBBI, Laura (2009) «Emerging Forms of Individualized Tourism in the Late Modernity. Tourism Tool of Cohesion in the Mediterranean Region ", DANS LATIESA RODRIGUEZ, Margarita; Inmaculada PUERTAS CANAVERAL et José L. PANIZA PRADOS (sous la direction de), El turismo en el Mediterráneo. Posibilidades de desarrollo y cohesión, p. 555-566. Madrid : Editorial Universitaria Ramón Areces.

GIOBBI, Laura (2010a) Per una sociologia delle mobilità: le nuove trame della società postmoderna, Milano : Franco Angeli. 139 p.

GIOBBI, Laura (2010b) Le nuove vacanze. Neoturismi, marketing pubblicitario e turismo di genere, Matera : Altrimedia Edizioni. 166 p.

GOFFMAN, Erving (1959) The presentation of self in everyday life, New York : Anchor Books. 244 p.

GOTTLIEB, A. (1982) «Americans' vacations», Annals of Tourism Research, vol. $9, \mathrm{n}^{\circ}$ 2, p. 165-187.

JAFARI, J. (1988) «Le système du touriste: modèles socio-culturels en vue d'applications théoriques et pratiques», Loisir et société, Sainte-Foy : Presses de l'Université du Québec, vol. 11, nº 1, p. 59-80.

JOWITT, Ken (1992) New world disorder: the Leninist extinction, Berkeley: University of California Press. 342 p.

KNAFOU, Remy (1998) Approche critique de la notion de "tourisme durable». Le cas des espaces marginaux et "extrêmes", DANS ZERBI, Maria Chiara (sous la direction de), Turismo sostenibile in ambienti fragili, Milano : Cisalpino. 599 p.

LYOTARD, Jean F. (1979) La condition postmoderne, Paris : Minuit. 128 p. MacCANNELL, Dean (1976) The tourist, New York: Shocken Books. 231 p. MAFFESOLI, Michel (1997) Du nomadisme. Vagabondages initiatiques, Paris : Librairie Générale Française. 206 p. 
NAESS, A. (1973) «The Shallow and the Deep, Long-Range Ecology

Movement: A Summary», Inquiry, nº 16, p. 95-100.

NOCIFORA, E. (sous la direction de) (1996) Il turismo contro l'integralismo, Napoli \& Los Angeles : Ipermedium. 163 p.

OMT - Organisation Mondiale du Tourisme (1999) Code mondial d'éthique du tourisme, OMT.

PARSONS, Talcott (1937) The structure of social action, New York : The Free Press. 817 p.

PEARCE, Douglas (1987) Tourism today: a geographical analysis, Harlow : Longman. 229 p.

SAVELLI, Asterio (1998) Sociologia del turismo, Milano : Franco Angeli. $376 \mathrm{p}$.

SAVELLI, Asterio (2004) Turismo, territorio, identità. Ricerche ed esperienze nell'area mediterranea, Milano : Franco Angeli. 359 p.

SEN, Amartya K. (1999) Development as freedom, Oxford : Oxford University Press. 366 p.

SHELLER, M. et John URRY (2006) «The new mobilities paradigm », Environment and Planning A, vol. 38, p. 207-226.
SMITH, V. L. (1977) Hosts and guests, the anthropology of tourism, Philadelphia : University of Pennsylvania Press. 254 p.

SWARBROOKE, John (1999) Sustainable tourism management, Wallingford : Cabi Publishing. 371 p.

UICN; PNUE et WWF - Union International pour la Conservation de la Nature; Programme des Nations Unies pour l'Environnement et Fond Mondial pour la Nature (1991) Caring for the Earth: a strategy for sustainable living, rapport, Gland, Suisse : UICN.

UNEP — Programme des Nations Unies pour l'environnement (1987) World Commission on Environment and Development, rapport Our Common Future, Royaume-Uni, Oxford : Oxford University Press. 300 p. URRY, John (1990) The tourist gaze: leisure and travel in contemporary societies, London : Sage. 183 p.

WARD, Colin et David HARDY (1986) Goodnight campers! The history of the British holiday camp, London : Mansell. 192 p.

\section{ANS D'EXPÉRIENCE EN TOURISME Complétez votre collection}

$\square$ Le tout-inclus vol. $31, n^{0} 2,2012$

$\square$ Gouvernance des parcs au Nunavik vol. $31, n^{0} 1,2012$

$\square$ Tourisme et patrimoine mondial Les associations de patrimoine et le tourisme vol. $30, n^{0} 2,2011$

$\square$ Excellence des destinations Ciné-tourisme vol. $30, n^{0} 1,2011$

$\square$ Géotourisme / Tourisme et femmes vol. 29, $n^{0} 2,2010$

$\square$ Tourisme des racines Tourisme et autochtones vol. $29, n^{0} 1,2010$

$\square$ Tourisme, sport et développement vol. $28, n^{0} 2,2009$

$\square$ Tourisme polaire vol. $28, n^{0} 1,2009$ $\square$ Nouveaux musées, nouveaux tourismes vol. $27, n^{0} 3$, automne 2008

$\square$ Les grands équipements touristiques vol. 27, n² 2, été 2008

$\square$ La science du tourisme vol. 27, $\mathrm{n}^{0} 1$, printemps 2008

$\square$ Tourisme et solidarité vol. 26, n³ 3 , automne 2007

$\square$ Tourisme et attractivité vol. 26, n 2, été 2007

$\square$ Tourisme Caraïbe vol. 26, $n^{0} 1$, printemps 2007

$\square$ Forêt vol. 25, n³, automne 2006

$\square$ Désirs d'Orient vol. 25, n² 2, été 2006

$\square$ Tourisme gourmand vol. 25, n 1 , printemps 2006 $\square$ Tourisme et santé vol. 24, n ${ }^{0} 3$, automne 2005

$\square$ Tourisme, religion et patrimoine vol. 24, n² 2, été 2005

$\square$ Maroc vol. 24, nº 1, printemps 2005

$\square$ Regards sur l'hôtellerie vol. 23, n 3, automne 2004

$\square$ La qualité en tourisme vol. 23, n² 2, été 2004

$\square$ Au risque du politique vol. 23, $n^{0} 1$, printemps 2004

$\square$ L'accessibilité, une conquête inachevée vol. 22, n 3, automne 2003

$\square$ Les routes à thèmes vol. 22, n’ 2, été 2003

$\square$ Tourisme et sexualité vol. 22, nº 1 , printemps 2003

\section{$20 \$$ / numéro, taxes incluses. Livraison gratuite pour tout achat de plus de $50 \$$.}

\section{Faites vos achats sur www.coopugam.com.}

